\title{
A novel ex-ante tariff scheme for cost recovery of transmission investments under elasticity of demand
}

\author{
Antonio De Paola ${ }^{1,2}$ \\ (1) REF-E S.r.l, Milan, Italy \\ ${ }^{(2)}$ Dept. of Electronic \& Electrical Eng. \\ University of Bath, Bath, UK \\ Email: a.de.paola@bath.ac.uk
}

Iacopo Savelli ${ }^{3}$

${ }^{(3)}$ Smith School of Enterprise and the Environment

University of Oxford, Oxford, UK

Email: iacopo.savelli@smithschool.ox.ac.uk

\author{
Thomas Morstyn ${ }^{4}$ \\ ${ }^{(4)}$ School of Engineering \\ University of Edinburgh, \\ Edinburgh, UK \\ Email: thomas.morstyn@ed.ac.uk
}

\begin{abstract}
This paper proposes the application of ex-ante dynamic network tariffs for the recovery of investment costs in transmission network planning. The tariffs design is integrated in the long-term network expansion problem and in the underlying market clearing process, accounting for the impact of tariffs on the bidding strategies of price-responsive participants. Differently from the established ex-post approach, this method avoids negative surplus for marginal users and untruthful biddings. The methodology is described analytically and applied to a relevant case study, which demonstrates the positive effect of ex-ante tariffs in terms of increased social welfare, emphasizing their positive impact in scenarios with high price-elasticity of demand.
\end{abstract}

Index Terms-Tariff design, network planning, bilevel optimization.

\section{INTRODUCTION}

The power grid is undergoing unprecedented changes as a result of the increasing penetration of renewables and the electrification of transportation and heating [1]. One of the most relevant transformations regards the development and planning of the network: $€ 114$ billion of investments on European infrastructure are envisaged by 2030 [2] to accommodate more variable renewable generation and changing demand patterns.

To maximize the positive impact of the network expansion on the social welfare of the system, it is crucial that the investment costs are distributed among the network stakeholders in a fair and efficient manner. This topic still presents several unresolved issues, regarding in particular the full and efficient recovery of the fixed component of the investments [3][7]. The current schemes for cost recovery vary significantly from country to country but they all share some fundamental features: i) they rely on a combination of congestion rent and network tariffs and ii) the tariffs are applied ex-post (after the market clearing), not taking into account their effect on pricesensitive consumers and producers [8]. This approach can lead to significant inefficiencies, in the form of negative surplus for marginal market participants and untruthful bidding [9]. These issues are expected to become even more important as new

This work was supported by the Engineering and Physical Sciences Research Council (award numbers EP/S031901/1 and EP/S000887/1). technologies and demand response mechanisms increase price elasticity on the demand side. Previous studies have tackled this issue by proposing new tariffs [10] and network charges [11] which account for the price-sensitivity of customers. However, these studies do not explicitly relate the tariff design with the network planning problem, nor do they consider the associated optimization complexities (e.g. lumpy investments).

This paper takes into account these additional elements by following the initial study of the authors [12] on a novel tariff scheme for investment cost recovery, termed ex-ante dynamic network tariffs. The analysis is extended to evaluate the performance of the mechanism in a context of increasing demand elasticity. With the proposed approach, the design of the tariffs is merged with the investment optimization and market clearing problem. This allows the effect of the tariffs on the market participants' behaviour to be considered explicitly, avoiding the aforementioned issues of negative surplus and untruthful bidding. The proposed framework envisages a bilevel formulation with two coupled optimizations: a longterm investment planning problem in the upper level and a market clearing problem in the lower level. The numerical resolution of the problem (recast as a mixed-integer linear program) is discussed, and a relevant case study is presented to highlight the advantages of the proposed approach in terms of increased efficiency and reduced negative surplus for consumers. Additional sensitivity analyses are conducted to demonstrate the capability of the tariff mechanism to accommodate higher elasticity of demand and avoid the increasing negative surplus for marginal users that would result from expost tariff approaches.

\section{TRANSMISSION NETWORK PLANNING}

The problem of expanding the electricity network in a cost-efficient manner is typically analysed under a centralized paradigm. With this approach, the lines to be expanded and their additional capacities are determined by a central planner, with the objective of maximizing the total social welfare [13]. 


\section{A. The network planning problem}

The centralized planning problem can be analytically represented by the following objective function:

$$
\begin{array}{r}
\max _{\substack{d_{t, k}, g_{t, p}, u_{m}, f_{t, m}, F_{m}}} \sum_{t \in \mathcal{T}}\left(\sum_{k \in \Omega_{t}^{D}} B_{k}\left(d_{t, k}\right)-\sum_{p \in \Omega_{t}^{G}} C_{p}\left(g_{t, p}\right)\right) \\
-\sum_{m \in \mathcal{M}} u_{m}\left(K_{m}^{f i x}+K_{m}^{v a r} F_{m}\right)
\end{array}
$$

where the social welfare is expressed as the difference over all time periods $t$ between i) the demand benefits $B_{k}$ minus the generation costs $C_{p}$ (over all consumers $k \in \Omega_{t}^{D}$ with demand $d_{t, k}$ and all generators $p \in \Omega_{t}^{G}$ with production $g_{t, p}$ ), and ii) the total investment costs. The planning decisions consist of the binary variables $u_{m}$, which indicate whether each line $m$ is expanded or not, and the continuous variables $F_{m}$, which determine the new additional capacities of the lines. The associated investment costs are the sum, over each expanded line $m$, of a fixed cost component $K_{m}^{f i x}$ and a variable term $K_{m}^{v a r} F_{m}$ (proportional to the additional capacity of the line). The optimization in (1) must fulfil the following constraints:

$$
\begin{gathered}
\sum_{k \in \Omega_{t, n}^{D}} d_{t, k}-\sum_{p \in \Omega_{t, n}^{G}} g_{t, p}+\sum_{m \in \mathcal{M}} a_{m, n} f_{t, m}=0 \quad \begin{array}{r}
\forall t \in \mathcal{T} \\
\forall n \in \mathcal{N}
\end{array} \\
\sum_{m \in \mathcal{M}} \psi_{\ell, m} f_{t, m}=0 \quad \forall t \in \mathcal{T}, \forall \ell \in \mathcal{L}
\end{gathered}
$$

where (2a) is the generation/demand balance for each network node $n$ (denoting by $a_{m, n}$ the branch-node incidence matrix [14]) and (2b) is the Kirchoff's voltage law evaluated over each network loop $\ell$. The term $\psi_{\ell, m}$ in (2b) is equal to the reactance of line $m$ if this belongs to the loop $\ell$ and is zero otherwise. Finally, the capacity limits of each line must be taken into account. Denoting by $F_{m}^{0}$ and $u_{m} F_{m}$ the initial and new capacity of line $m$, respectively, the following must hold for the power flow $f_{t, m}$ :

$-u_{m} F_{m}-F_{m}^{0} \leq f_{t, m} \leq F_{m}^{0}+u_{m} F_{m} \quad \forall t \in \mathcal{T}, \forall m \in \mathcal{M}$.

Note that the solution of (1), subject to (2)-(3), not only returns the line expansion decisions $u_{m}$ and $F_{m}$ but it also determines the demand levels $d$, the generation profiles $g$ and line flows $f$ that correspond to the maximized social welfare in the expanded network. Under the classical assumptions of perfect competition (price-taker participants, perfect knowledge, no discrimination), the triplet $(d, g, f)$ returned by (1) can alternatively be interpreted as the solution of a market-clearing problem in the expanded network, where each demand and generation unit bids according to its marginal utility or cost.

\section{B. Investment cost recovery}

Within the considered centralized paradigm, the investment costs sustained to expand the network must be smaller than or equal to the associated increase in social welfare, ensuring a net benefit for the system. However, a fair and efficient distribution of the costs among the different system agents remains an open problem. In the planning problem (1), the investment costs can be recovered through the congestion rent (CR) collected on the transmission lines. The congestion rent $C R_{m}$ of a certain line $m$ corresponds to the product, over all time periods $t$, between i) the flow $f_{t, m}$ on the line, and ii) the difference of the locational marginal price $\pi_{t, n}$ between the receiving node $n_{m}^{r}$ and the sending node $n_{m}^{s}$ :

$$
C R_{m}=\sum_{t \in \mathcal{T}}\left(\pi_{t, n_{m}^{r}}-\pi_{t, n_{m}^{s}}\right) f_{t, m}
$$

The revenue adequacy condition of the investment can therefore be expressed as:

$$
C R=\sum_{m \in \mathcal{M}} C R_{m} \geq \sum_{m \in \mathcal{M}} u_{m}\left(K_{m}^{f i x}+K_{m}^{v a r} F_{m}\right) .
$$

where the right-hand side denotes the total investment costs. The congestion rent CR can always ensure a full recovery of the variable costs [13]. However, there is no guarantee that it will also cover the fixed cost components. This means that, if the central planner (e.g. the transmission system operator) is only entitled to collect the congestion rent, the fixed costs $K_{m}^{f i x}$ associated to the expanded lines cannot be recovered, despite the fact that the expansion actually generates sufficient welfare to cover all the costs.

For this reason, additional mechanisms are generally introduced to recover the remaining investment costs from network users, which have benefited from the increased social welfare delivered by the investment. This has been usually achieved through ex-post tariffs, i.e. additional payments from generation and demand after the market clearing. However, this solution presents relevant drawbacks and can lead to suboptimal solutions, as demand and supply curves in dayahead markets are price-elastic [15]. That is, consumers and producers trade different amounts of energy at different prices. For example, at higher prices, generators will be willing to supply more energy whereas consumers will tend to buy less. For these price-sensitive market participants, any additional cost applied after the market clearing (e.g. ex-post network tariffs) can lead to a negative surplus. In particular, generators and consumers that are trading at their marginal cost/benefit after the market clearing, will become out-of-the-money when the additional ex-post tariffs are applied. This problem is becoming more relevant on the buyers side, as technological developments and demand response schemes are increasing the flexibility and price-elasticity of demand.

\section{EX-ANTE NETWORK TARIFFS}

The approach presented in this paper overcomes the drawbacks detailed above by introducing a novel cost-recovery scheme that relies on ex-ante network tariffs, i.e. tariffs that are included in the market clearing process and are related to the traded quantities. Under the proposed framework, the total cost of the generator $p$ is made up of two components: the original generation cost $\widetilde{C}_{p}$ and the additional network 
charging $T_{p}$. The resulting marginal cost at the time period $t$ can then be expressed as:

$$
p_{t, p}^{G}\left(g_{t, p}\right)=\frac{\partial \widetilde{C}_{p}\left(g_{t, p}\right)}{\partial g_{t, p}}+\frac{\partial \mathrm{T}_{p}\left(g_{t, p}\right)}{\partial g_{t, p}} .
$$

Similarly, for any consumer $k$, the original benefit function $\widetilde{B}_{k}$ will be reduced by the network charge $\mathrm{T}_{k}$. The resulting marginal benefit $p_{t, k}^{D}$ at time $t$ is the following:

$$
p_{t, k}^{D}\left(d_{t, k}\right)=\frac{\partial \widetilde{B}_{k}\left(d_{t, k}\right)}{\partial d_{t, k}}-\frac{\partial \mathrm{T}_{k}\left(d_{t, k}\right)}{\partial d_{t, k}} .
$$

In the chosen formulation, the charged network costs $\mathrm{T}_{p}$ and $\mathrm{T}_{k}$ correspond to the product (over each relevant line $m$ ) between the tariff $\tau_{m}$ and the generation $g$ and demand $d$, respectively:

$$
\mathrm{T}_{p}\left(g_{t, p}\right):=\sum_{m \in \mathcal{M}} \tau_{m} g_{t, p}, \quad \mathrm{~T}_{k}\left(d_{t, k}\right):=\sum_{m \in \mathcal{M}} \tau_{m} d_{t, k} .
$$

The marginal cost and benefit in (6)-(7) can therefore be recast as:

$$
p_{t, p}^{G}=\widetilde{p}_{t, p}^{G}+\sum_{m \in \mathcal{M}} \tau_{m}, \quad \quad p_{t, k}^{D}=\widetilde{p}_{t, k}^{D}-\sum_{m \in \mathcal{M}} \tau_{m} .
$$

The fundamental feature of the formulation in (6)-(9) is the possibility to dynamically consider the effect of the network tariffs on the bidding strategies of producers and consumers, and on the associated market clearing, avoiding the sub-optimality effects of ex-post network tariffs discussed in Section II-B. The additional terms $\mathrm{T}_{p}$ and $\mathrm{T}_{k}$ will increase the bidding profile of generators (as they need to cover the additional network costs) and lower the bids of consumers (as their benefit is reduced by the additional charges). As depicted in the example in Figure 1, ex-ante tariffs will generally lead to a market clearing solution with different prices (from $p_{1}$ to $p_{2}$ ) and volumes (from $q_{1}$ to $q_{2}$ ). The reduction in the cleared quantity relates to the price-sensitive participants who would suffer a loss if tariffs were applied ex-post to the original volume $q_{1}$.

\section{NETWORK PLANNING WITH EX-ANTE TARIFFS}

The key feature of the proposed scheme is its capability to analytically embed network costs (e.g. tariffs) into participants' bid profiles, accounting for their impact on the cleared volumes and market prices. These quantities affect in turn the congestion rent collected by the transmission system operator and ultimately the selected tariffs, which are determined to ensure the recovery of the overall investment costs. Since the exchanged volumes, the market prices, the network costs and the collected congestion rent are dynamically linked when exante tariffs are considered, it is necessary to develop a novel network planning problem that considers all these quantities simultaneously. To this end, a bilevel optimization framework is proposed, as summarized in Fig. 2.



Fig. 1: Supply curve (red) and demand curve (blue) before network costs (dashed lines) and after the application of a 5 f/MW network tariff (solid lines). The tariff shifts the demand curve downwards and the supply curve upwards, changing the clearing price and volumes from $\left(p_{1}, q_{1}\right)$ to $\left(p_{2}, q_{2}\right)$.

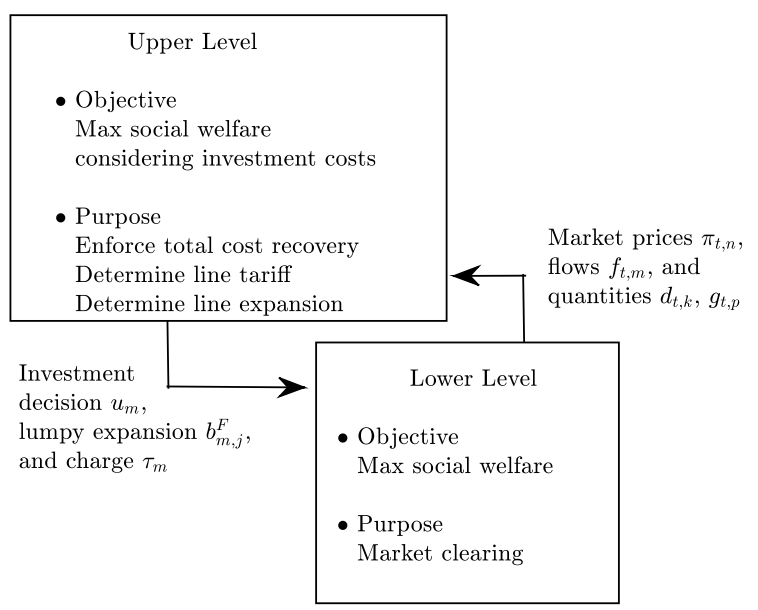

Fig. 2: Bilevel formulation of the network planning problem.

\section{A. Upper level optimization}

The upper level program corresponds to the long term network planning problem. On the basis of the market clearing solution $(\pi, f, d, g)$ obtained from the lower level problem, the upper level returns the line expansions $u_{m}$ and the network tariffs $\tau_{m}$ that maximize the overall social welfare while ensuring full recovery of the investment costs (both fixed and variable components). In this framework, the objective function is the following:

$$
\begin{aligned}
\max _{\begin{array}{c}
u_{m}, b_{m, j}^{F}, \tau_{m} \\
p_{t, k}^{D}, p_{t, p}^{G}
\end{array}} \sum_{t \in \mathcal{T}}\left(\sum_{k \in \Omega_{t}^{D}} \widetilde{p}_{t, k}^{D} d_{t, k}-\sum_{p \in \Omega_{t}^{G}} \widetilde{p}_{t, p}^{G} g_{t, p}\right) \\
-\sum_{m \in \mathcal{M}}\left(u_{m} K_{m}^{f i x}+K_{m}^{v a r} \sum_{j \in \mathcal{J}} b_{m, j}^{F} \bar{F}_{m, j}\right) .
\end{aligned}
$$

Note that (10) represents the overall social welfare of the system as in the initial formulation in (1), with some small differences. In this case, the decision variables include the binary variables $b_{m, j}^{F}$ and the (positive) tariff parameters $\tau_{m}$. The former are used to account for lumpy investments and select a specific value of additional capacity among the set of 
discretised feasible expansions options $\overline{\mathcal{F}}_{m}=\bigcup_{j \in \mathcal{J}} \bar{F}_{m, j}$. The uniqueness of the selection on each line $m$ is guaranteed by the condition $\sum_{j \in \mathcal{J}} b_{m, j}^{F}=u_{m}$. The parameters $\tau_{m}$ are used to implement the tariff scheme introduced in (6)-(9) through the following constraints:

$$
\begin{aligned}
& p_{t, k}^{D}=\widetilde{p}_{t, k}^{D}-\sum_{m \in \mathcal{M}} u_{m} \tau_{m} \quad \forall t \in \mathcal{T}, \forall n \in \mathcal{N}, \forall k \in \Omega_{t, n}^{D} \\
& p_{t, p}^{G}=\widetilde{p}_{t, p}^{G}+\sum_{m \in \mathcal{M}} u_{m} \tau_{m} \quad \forall t \in \mathcal{T}, \forall n \in \mathcal{N}, \forall p \in \Omega_{t, n}^{G} .
\end{aligned}
$$

The quantities $\tau_{m}$ do not appear explicitly in (10), since the charging costs are paid by consumers and producers and are collected by the central planner, resulting in a zero variation of the overall welfare. In this formulation, the additional revenue adequacy condition to be fulfilled by the selected tariffs is:

$$
\begin{aligned}
& \sum_{t \in \mathcal{T}} \sum_{m \in \mathcal{M}} \sum_{n \in \mathcal{N}}-a_{m, n} \pi_{t, n} f_{t, m}+ \\
& \sum_{t \in \mathcal{T}} \sum_{m \in \mathcal{M}} \sum_{n \in \mathcal{N}} u_{m} \tau_{m}\left(\sum_{k \in \Omega_{t, n}^{D}} d_{t, k}+\sum_{p \in \Omega_{t, n}^{G}} g_{t, p}\right) \geq \\
& \sum_{m \in \mathcal{M}}\left(u_{m} K_{m}^{f i x}+K_{m}^{v a r} \sum_{j \in \mathcal{J}} b_{m, j}^{F} \bar{F}_{m, j}\right) .
\end{aligned}
$$

Condition (12) imposes that the revenues collected by the central planner (i.e. congestion rent plus network charging payments from consumers and generators) are greater or equal than the total investment costs.

\section{B. Lower level optimization}

The lower level of the bilevel formulation corresponds to a standard market clearing problem that considers the network expansion determined in the upper level and accounts for the changes introduced by the tariff scheme on the bid functions of generators and consumers. Assuming no time-coupling constraints and considering linear functions for demand benefits and generation costs, a unique clearing problem can be formulated over all the considered time periods, with the following objective function:

$$
\max _{d_{t, k}, g_{t, p}, f_{t, m}} \sum_{t \in \mathcal{T}} \sum_{n \in \mathcal{N}}\left(\sum_{k \in \Omega_{t, n}^{D}} p_{t, k}^{D} d_{t, k}-\sum_{p \in \Omega_{t, n}^{G}} p_{t, p}^{G} g_{t, p}\right) .
$$

The optimization in (13) returns the generation schedule $g$, the demand levels $d$ and the resulting power flows $f$ that correspond to a maximized social welfare. The impact of the tariffs, selected in the upper level, is taken into account through the quantities $p^{D}$ and $p^{G}$, as defined in (11a) and (11b), respectively. In addition to the constraints (2) of the initial formulation, the capacity limits of the lines (accounting for the lumpy expansions $\bar{F}_{m, j}$ determined in the upper level) are characterized as follows (for all $t \in \mathcal{T}$ and $\forall m \in \mathcal{M}$ ):

$$
-F_{m}^{0}-\sum_{j \in \mathcal{J}} b_{m, j}^{F} \bar{F}_{m, j} \leq f_{t, m} \leq F_{m}^{0}+\sum_{j \in \mathcal{J}} b_{m, j}^{F} \bar{F}_{m, j}
$$

\section{Resolution of the planning problem}

The bilevel optimization framework summarized in Fig. 2 and described by (10)-(14) can in principle be solved by iterated resolutions of its two subproblems, until convergence to equilibrium. However, in order to avoid potential issues of convergence and computational time of this approach, the bilevel formulation is recast as a single nonlinear problem which, after some algebraic manipulations to remove nonlinearities, is characterised as a standard MILP problem that can be solved with off-the-shelf solver. The general idea is to represent the lower level problem by its equivalent KKT conditions, which are then considered as additional constraints of the upper level. In particular, the primal and dual constraints of the lower level are included, while the standard slackness condition is replaced by the strong duality property. The resulting nonlinear optimization problem is then formulated as a MILP by applying complementarity relations and standard algebraic manipulations. A detailed description of the procedure can be found in [12].

\section{RESUlts AND DISCUSSION}

This section presents relevant case studies to highlight the main features of the proposed ex-ante dynamic network tariffs. In particular, it is shown that this novel approach can overcome the issues of negative surplus for marginal users exhibited by ex-post approaches. Moreover, the impact of an increasing demand elasticity on the optimal network planning and on the resulting tariffs is evaluated. The analysis considers the following scenarios:

(i) No-Tariff Solution (NTS): the basic planning approach defined in (1) - (3).

(ii) No-Tariff Solution with revenue adequacy (NTR): extension of NTS that also enforces the revenue adequacy condition (5).

(iii) No-Tariff Solution with revenue adequacy and lumpy expansion (NTR-L): NTR with lumpy expansions. Line capacities can be increased only by discrete amounts (equal to $3 \mathrm{MW}$ in this case study).

(iv) Ex-Ante Tariff Solution (ETS): the proposed ex-ante dynamic network tariff scheme introduced in Section IV.

In order to highlight the fundamental features of the proposed approach, the 2-bus network in Figure 3 is analysed (the interested reader is referred to [12] for the analysis of different configurations).

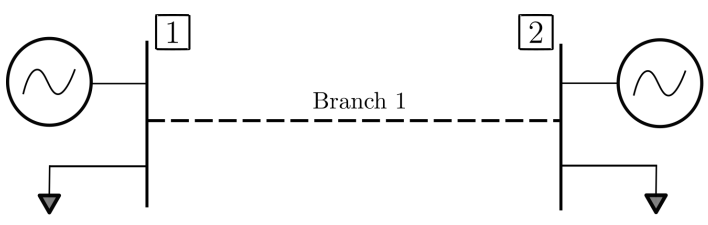

Fig. 3: Topology of the 2-node system.

In this scenario, the problem is to determine the optimal expansion for Branch 1 connecting the two nodes, as well as the optimal tariff $\tau_{m}$ levied on the network users, while 



Fig. 4: Demand (blue) and supply (red) curves in node 1 (right) and node 2 (left).

ensuring the recovery of the overall investment costs. The fixed cost $K_{m}^{f i x}$ is equal to $100 £$, whereas the variable cost $K_{m}^{v a r}$ is equal to $10 £ / \mathrm{MW}$. The demand and supply curves at each node are depicted in Figure 4. Having fixed the relevant parameters of the case study, the associated economic quantities to be considered in the planning problem are compared in Fig. 5. In the figure, the areas $C R_{1}$ and $C R_{2}$ represents the congestion revenues that ensure the recovery of variable and total investment costs, respectively. The term $\Delta \pi$ is the price differential between the two nodes resulting from the expansion $F_{m}$ which, in this example with pre-existing line capacity $F_{m}^{0}=0 \mathrm{MW}$, is equal to the power flow $f_{m}$ on the line. The quantity $C_{a v}$ indicates the cost per unit of additional capacity (as a function of the expansion $F_{m}$ ) and it is expressed as:

$$
C_{a v}\left(F_{m}\right)=\frac{K_{m}^{f i x}+K_{m}^{v a r} F_{m}}{F_{m}}=\frac{K_{m}^{f i x}}{F_{m}}+K_{m}^{v a r} .
$$

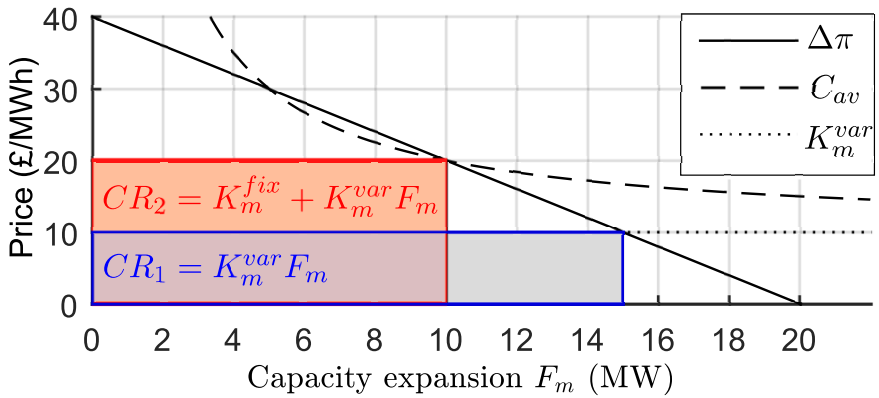

Fig. 5: Relevant economic quantities of the expansion problem.

The network expansion and associated cost recovery for the 2-node system have been evaluated according to the four approaches detailed at the beginning of this section and the results are reported in Table I. In the NTS model (1)-(3), the

TABLE I: Results of the planning models for the 2-node system.

\begin{tabular}{|c|c|c|c|c|r|}
\hline $\begin{array}{c}\text { Planning } \\
\text { model }\end{array}$ & $\begin{array}{c}\text { Line } \\
\text { exp. } \boldsymbol{F}_{\boldsymbol{m}} \\
(\mathbf{M W})\end{array}$ & $\begin{array}{c}\text { Social } \\
\text { welfare } \\
(\mathfrak{E})\end{array}$ & $\begin{array}{c}\text { Total } \\
\text { costs } \\
(\mathfrak{f})\end{array}$ & $\begin{array}{c}\text { Cong. } \\
\text { rent } \\
(\mathfrak{E})\end{array}$ & $\begin{array}{c}\text { Tariff } \\
\boldsymbol{\tau}_{\boldsymbol{m}} \\
(\mathfrak{f} / \mathbf{M W h})\end{array}$ \\
\hline NTS & 15 & 2125 & 250 & 150 & 0 \\
\hline NTR & 10 & 2100 & 200 & 200 & 0 \\
\hline NTR-L & 9 & 2089 & 190 & 198 & 0 \\
\hline ETS & 12 & 2115 & 220 & 189 & 0.353 \\
\hline
\end{tabular}

revenue adequacy constraint (5) is not enforced. In this case, the optimal solution is to have $F_{m}=15 \mathrm{MW}$ of additional capacity on line 1 , for a total investment of $250 £$ (100£ of fixed costs plus $150 £$ of variable costs). This solution corresponds to the intersection between $K_{m}^{v a r}$ and $\Delta \pi$, implying that, in the NTS, the congestion rent $C R_{1}=\Delta \pi F_{m}$ is exactly equal to the variable costs $K_{m}^{v a r} F_{m}$. It follows that the congestion revenues cannot ensure the recovery of the fixed costs, leading to a net loss of $100 £$ for the network investor which would need to be recovered through ex-post tariffs. This means that the associated social welfare of $2125 £$ is not achievable, as it would lead to a negative surplus for the marginal market participants.

To overcome this issue, the revenue adequacy constraint (5) can be enforced, leading to the NTR scheme. In this model, the only source of revenue for cost recovery of the investment remains the congestion rent. To ensure that this quantity covers not only the variable costs of the investment but also the fixed costs, the network investor has to reduce the line capacity (i.e. underinvest) while reaching a higher price differential $\Delta \pi$ that increases the congestion rent. In this case, the optimal solution is the capacity $F_{m}=10 \mathrm{MW}$ for Branch 1 , which corresponds to the intersection between $\Delta \pi$ and $C_{a v}$ in Figure 5. It follows that the red area representing the congestion revenues $C R_{2}$ is also equal to the total investment cost, i.e. the product between the per-unit cost $C_{a v}$ and the additional capacity $F_{m}$. Note that the NTR, by considering a smaller capacity expansion with respect to the NTS, achieves an overall social welfare of $2100 £$, which is lower than the $2125 £$ obtained in the NTS.

The inefficiency of the expansions is further exacerbated in the NTR-L model with lumpy expansions, where it is assumed that the line capacity can be increased only by discrete steps of $3 \mathrm{MW}$. It follows that the capacity expansion $F_{m}=10 \mathrm{MW}$ of the NTR is not feasible in the NTR-L and the network planner has to further underinvest, leading to a line expansion of $9 \mathrm{MW}$. Observing Figure 5, for an expansion of $F_{m}=$ $9 \mathrm{MW}$ the average total costs $C_{a v}$ are strictly lower than the price difference $\Delta \pi$. Equivalently, the total investment costs $(190 £)$ are strictly smaller than the collected CR (198 £), leading to a strictly positive profit for the investor $(8 £)$ and a further reduction of the social welfare (down to $2089 £$ ).

By contrast, when the proposed ETS model (with ex-ante dynamic network tariffs) is considered, the lumpy expansion of $12 \mathrm{MW}$ is achievable. In this case, the recovery of the total investment costs $(220 £)$ is repartitioned, in an optimal manner,

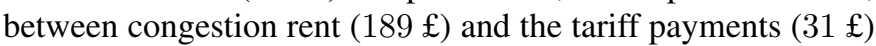
collected with the tariff $\tau_{m}=0.353 \mathrm{f} / \mathrm{MWh}$. This leads to a significant social welfare improvement with respect to the NTR and NTR-L cases, while still ensuring full recovery of the investment costs. This example shows how the introduction of network tariffs can lead to improved efficiency for the system, despite the tariff payments. When the only source of revenues for the investor is the congestion rent, an underinvestment is necessary to recover the fixed costs (from $15 \mathrm{MW}$ to $9 \mathrm{MW}$ in the example). Conversely, network tariffs allow network users to directly compensate the operator for its fixed costs, while achieving a higher social welfare. 
It has been emphasized in previous sections that the current ex-post network tariffs can lead to negative surplus for some customers and this issue is exacerbated by increasing priceelasticity of demand. To quantify this phenomenon and assess the potential benefits of the proposed ETS scheme, a sensitivity study is performed on demand elasticity. In addition to the base case presented in Fig. 4, we consider two additional scenarios termed elastic and inelastic. We recall that for a linear demand bid curve $p_{n}=a_{n}-b_{n} \times q_{n}$, where $p_{n}$ is the price and $q_{n}$ the traded volume in node $n$, the price-elasticity of demand is equal (in absolute value) to: $\epsilon_{n}=p_{n} /\left(b_{n} q_{n}\right)$. In the base case in Fig. 4, the demand curves (blue) correspond to $b_{1}=4$ (node 1 ) and $b_{2}=2$ (node 2). The demand curves in the elastic (inelastic) scenarios have been obtained through a $10 \%$ decrease (increase) of these initial values, adjusting the parameter $a_{n}$ to keep the total cleared quantity comparable. The impact of the elasticity on the results of the ETS model is summarized in Table II.

TABLE II: ETS model with different demand curve elasticity

\begin{tabular}{|c|r|r|r|r|}
\hline $\begin{array}{c}\text { ETS model } \\
\text { demand }\end{array}$ & $\begin{array}{c}\text { Cong. } \\
\text { rent (£) }\end{array}$ & $\begin{array}{c}\text { Tariff } \\
\boldsymbol{\tau}_{\boldsymbol{m}} \\
(\mathfrak{f} / \mathbf{M W h})\end{array}$ & $\begin{array}{c}\text { Neg. dem. } \\
\text { surplus } \\
\text { ex-post }\end{array}$ & $\begin{array}{c}\text { Neg. dem. } \\
\text { surplus } \\
\text { ex-ante }\end{array}$ \\
\hline Elastic & 214 & 0.068 & $2.55 \%$ & 0 \\
\hline Base & 189 & 0.353 & $2.46 \%$ & 0 \\
\hline Inelastic & 164 & 0.670 & $2.39 \%$ & 0 \\
\hline
\end{tabular}

In all three cases, the line is expanded by $12 \mathrm{MW}$, for a total cost of $220 £$. Despite the same line expansion, the change of the demand elasticity has a significant effect on the network tariff, which increases in the inelastic case to compensate for the lower congestion rent. As initially discussed, an ex-post application of the tariffs leads to a non-negligible percentage of volumes being traded with negative surplus (i.e. at a loss). In all cases, the ex-ante tariff scheme is able to reduce this quantity to zero. Note that the positive impact of the proposed approach becomes more substantial in the case of elastic demand, when the ex-post negative surplus is bigger (2.55\%).

\section{CONCLUSION}

The existing electrical network will require significant investments in order to cope with increasing demand and deployment of renewable energy sources. For this reason, it is of paramount importance to address open issues in network expansion planning, including i) the recovery of fixed costs, ii) negative surplus of marginal users when tariffs are applied after market clearing, i.e. ex-post, iii) lumpy expansions. The proposed framework tackles these timely issues by introducing ex-ante dynamic network tariffs, whose impact on the market clearing process with price-elastic participants is explicitly taken into account. In turn, the market clearing determines the congestion rent collected by the network operator, which is therefore indirectly affected by the network tariff, leading to a dynamic selection process. The analysis and the numerical studies presented in this paper demonstrate that ex-ante tariffs can be designed in order to fully recover investment costs (including their fixed component) through an optimized mix of congestion revenues and tariff payments. Moreover, by considering tariffs directly into the clearing process, the proposed approach ensures that the surplus of all users is always non-negative, regardless of the network costs charged. Finally, the monetary compensation introduced by the ex-ante tariffs allows to considers lumpy expansions that could not be supported exclusively by the congestion rent. Further work will extend the proposed framework to consider different tariff allocations between consumers and producers, performing a detailed comparison between the proposed design and existing tariff schemes (e.g. flow-based MW-mile methods).

\section{REFERENCES}

[1] Global energy transformation - roadmap to 2050, Tech. rep., IRENA (2019).

URL https://www.irena.org/DigitalArticles/2019/Apr/-/media/ 652AE07BBAAC407ABD1D45F6BBA8494B.ashx

[2] Ten years network development plan, Tech. rep., ENTSO-E (2018). URL https://tyndp.entsoe.eu/tyndp2018/

[3] F. P. Ramsey, A contribution to the theory of taxation, The Economic Journal 37 (145) (1927) 47-61.

[4] M. Boiteux, Peak-load pricing, The Journal of Business 33 (2) (1960) 157-179.

[5] M. Boiteux, On the management of public monopolies subject to budgetary constraints, Journal of economic Theory 3 (3) (1971) 219240.

[6] T. Kishore, S. Singal, Analysis of investment issues and transmission schemes for grid integration of remote renewable energy sources, International Journal of Renewable Energy Research (IJRER) 5 (2) (2015) 483-490.

[7] S. Borenstein, J. R. Bushnell, Do two electricity pricing wrongs make a right? cost recovery, externalities, and efficiency, in: The National Bureau of Economic Research, Working paper n. 24756, 2018. doi: $10.3386 / w 24756$.

[8] NETS, Connection and use of system code (2019).

URL www.nationalgrideso.com/codes/connection-and-use-systemcode-cusc

[9] J. Bushnell, Efficiency and cost recovery for transmission network investments, Department of Economics, University of California (Mar. 2014).

URL https://www.ea.govt.nz/dmsdocument/17782

[10] T. Schittekatte, I. Momber, L. Meeus, Future-proof tariff design: Recovering sunk grid costs in a world where consumers are pushing back, Energy Economics 70 (2018) 484 - 498. doi:https://doi.org/10.1016/j.eneco.2018.01.028.

[11] I. Abdelmotteleb, T. Gómez, J. P. C. Ávila, J. Reneses, Designing efficient distribution network charges in the context of active customers, Applied Energy 210 (2018) 815 - 826. doi:https://doi.org/10.1016/j.apenergy.2017.08.103.

[12] I. Savelli, A. De Paola, F. Li, Ex-ante dynamic network tariffs for transmission cost recovery, Applied Energy 258 (2020) 113979. doi:https://doi.org/10.1016/j.apenergy.2019.113979.

[13] D. S. Kirschen, G. Strbac, Fundamentals of power system economics, John Wiley \& Sons, 2004.

[14] D. R. Biggar, M. R. Hesamzadeh, The economics of electricity markets, John Wiley \& Sons, Chichester, UK, 2014.

[15] C. Su, D. Kirschen, Quantifying the effect of demand response on electricity markets, IEEE Transactions on Power Systems 24 (3) (2009) 1199-1207. doi:10.1109/TPWRS.2009.2023259. 